\title{
Quality of life in patients with bullous dermatoses
}

\author{
Deshan F. Sebaratnam, MBBS ${ }^{a}$, James R. McMillan, PhD $^{b}$, Victoria P. Werth, MD $^{\mathrm{c}}$, and \\ Dédée F. Murrell, MA, BMBCh, MD ${ }^{a}{ }^{*}$ \\ aDepartment of Dermatology, St. George Hospital, University of New South Wales, Grey Street, \\ Kogarah, NSW 2217, Australia \\ bCentre for Children's Burns and Trauma Research (CCBTR), Queensland Children's Medical \\ Research Institute (QCMRI), L/4 RCH Foundation Building, Royal Children's Hospital, The \\ University of Queensland, Herston, Brisbane, Queensland, 4029, Australia \\ 'Department of Dermatology, University of Pennsylvania School of Medicine, 2 Rhoads Pavilion, \\ 3600 Spruce Street, Philadelphia, PA 19104, USA
}

\begin{abstract}
Genetic and acquired bullous dermatoses can severely affect multiple domains of a patient's quality of life (QOL). Integrating formal evaluation of QOL into the clinical evaluation of patients facilitates an objective assessment of disease severity, mapping of disease trajectory, and captures therapeutic intervention outcomes. There have been 5 studies evaluating QOL in autoimmune dermatoses and 4 studies reviewing QOL in the genodermatoses. All literature to date indicates a significant disease burden in this setting. The development of formal QOL instruments has facilitated quantification of QOL deficits in this arena and offers promising tools for patient assessment in the future.
\end{abstract}

\section{Introduction}

Quality of life (QOL) has been defined as "the individual's perception of their position in life, in the context of the cultural and value systems in which they live and in relation to their goals, expectations, standards and concerns." (p1405) It is a multifaceted construct shaped by health as well as non-medical variables such as personality, ambition, expectation, socioeconomic and marital status, and religious experience. ${ }^{2}$ Many dermatological conditions exist, which pose minimal threat to patients in terms of mortality, but have the capacity to severely impinge on a patient's QOL. For instance, patients with psoriasis have reported a reduction in physical and mental functioning comparable to patients with cancer, arthritis, and depression. ${ }^{3}$ This is often due to the disfiguring nature of skin pathology with its adverse impact on body image and succeeding burden on social function, which may be independent of clinical severity. Symptoms, such as itch and pain, can strongly affect life quality and furthermore auxiliary considerations, such as functional limitations, financial burden, and possible side effects of treatments, all exert a detrimental effect on QOL.

Accordingly, an assessment of QOL provides a suitable means to evaluate the impact of a patient's dermatosis on their well-being and quantifying this impact has been a relatively recent effort stemming from a desire to evaluate the outcomes of medical intervention. ${ }^{4}$ Several QOL measurement tools have been developed as a means to evaluate the impact of

(C) 2012 Elsevier Inc. All rights reserved.

"Corresponding author. Tel.: +61 29113 2543; fax: +61 29113 2906. d.murrell@unsw.edu.au (D.F. Murrell). 
disease on patient well-being in a reliable, statistically validated way. The literature puts forward that patients and dermatologists often have different perceptions of a patient's QOL and that this discrepancy is associated with lower satisfaction with medical care. ${ }^{5}$ The measurements derived from QOL tools can be used alongside clinical assessment and disease activity measurements objectively to score a patient's disease severity and some tools allow analysis of which aspects of a patient's disease affects them the most (eg, symptoms such as itch or pain, the cosmetic insult, financial burden) facilitating the provision of personalized care. Evaluation of QOL can also be used to assess the effectiveness of therapeutic interventions and monitor disease trajectory over time, as well as providing a promising patient-based end point for clinical trials to quantify changes in disease process that may not correlate with changes in clinical disease activity. ${ }^{2}$ Quantifying the burden of disease is also important due to its implications for patient advocacy relating to the allocation of research support and clinical resources as well as for public perception of dermatological conditions. ${ }^{6}$

\section{Quality-of-life assessment techniques}

A variety of instruments and psychometric techniques exist to assess QOL in the clinical setting, and these can be categorized as generic, skin-specific, or disease-specific. Generic QOL measures have been used by the greater medical community to assess life quality across general medical problems. The advantages of generic instruments include their breadth and applicability across different conditions and interventions; however, adequate assessment of QOL necessitates instruments that are capable of capturing any changes, however small, that affect the patient. ${ }^{7}$ Generic QOL instruments do not possess this responsiveness but are often used because they provide a suitable means to compare cohorts across the greater community. Many skin-specific QOL instruments have also been developed and have the advantage of being more sensitive and responsive compared with generic measures. Although generic measures have been tested and validated repeatedly for their psychometric properties, it has been proposed that they do not necessarily capture issues that are specific to patients with skin conditions. ${ }^{4}$ Dermatology-specific instruments generally explore a particular domain in greater depth than the corresponding domain of a generic measure, enabling a heightened responsiveness to any changes that may occur, ${ }^{7}$ thus allowing the comparison of patients with different skin conditions within dermatology. Disease-specific QOL instruments are even more sensitive than skin-sensitive tools, as they have the capacity to capture specific issues associated with a particular disease. The advantage of these disease-specific instruments is that the questions are perceived to be of direct relevance to the patients, having a high face validity, and that the specificity of the questions maximizes the ability to detect any changes in disease process ${ }^{8}$; however, investigators cannot apply more specific instruments across diverse populations or different conditions, making comparisons between groups difficult. As such, researchers often use a combination of specific and generic QOL instruments to obtain satisfactory responsiveness and comparability. Many instruments also offer scales exploring particular domains within QOL, for instance the Medical Outcome Study 36-item Short-Form Survey (SF-36) ${ }^{9}$ is a general QOL tool divided into dimensions, including physical functioning, general health, vitality, social functioning, role-emotional, and mental health, that can be used to further explore QOL in greater detail.

\section{Literature on quality of life in autoimmune blistering diseases}

The detrimental impact of bullous dermatoses on QOL has been evaluated in the literature. Studies relating to QOL in autoimmune bullous dermatoses have primarily focused on pemphigus, namely pemphigus vulgaris, and there exists a paucity in the literature regarding QOL in other forms of pemphigus as well as the other acquired bullous diseases, such as 
bullous pemphigoid, mucous membrane pemphigoid, epidermolysis bullosa aquisita, and linear IgA bullous dermatosis.

The earliest study exploring QOL in autoimmune blistering disease (AIBD) involved 380 pemphigus patients in Japan. ${ }^{10}$ Patients were stratified as having mild (67.7\%), moderate (25.9\%), or severe $(6.3 \%)$ disease and asked to complete a survey reviewing their activities of daily living (ADLs) and the financial impact of their condition. Patients were found to be independent in most ADLs, however experienced a considerable economic burden because of their disease. In the cohort, $41.9 \%$ described a decrease in income owing to lost work time $(60.0 \%$ in severe disease) and $41.3 \%$ described their financial status as being "in poverty" ( $57.9 \%$ in severe disease). Only $2.2 \%$ of patients were in the active stage of disease with the remainder in varying phases of remission, and accordingly the results illustrated that with appropriate treatment pemphigus does not significantly impinge on the ADLs of patients. In contrast, the financial impact of pemphigus may be onerous even if the patient shows clinical improvement, and this should be considered in the assessment of these patients.

Another study evaluating QOL in pemphigus was conducted using formal QOL instruments - the SF-36 - to compare QOL in patients with pemphigus and the greater population in Morocco. ${ }^{11}$ The study cohort included 30 consecutive patients with pemphigus presenting at the dermatology clinic or admitted to the hospital, and the control cohort consisted of 60 individuals presenting with non-debilitating problems such as verruca. The SF-36 was translated into the Moroccan dialect, and as there is a high illiteracy rate in Morocco, the questionnaires were administered by a single investigator, rather than being patient administered. The authors identified a significant difference between cohorts with the pemphigus group displaying decreased mean scores across all dimensions except for "physical pain" and "change compared with the previous year." Physical and emotional status were the areas most affected by pemphigus, with facial involvement and the extent of lesions correlating well with overall scores. Within the group, $70 \%$ expressed "enormous shame" about their appearance, $60 \%$ were anxious about what others thought of their disease, and $63 \%$ reported a significant loss of confidence $(n=30)$. Repercussions for sexual function were also important, with $81 \%$ of patients reporting concerns in this domain. The authors noted that social factors had an important role to play in this setting, as misconceptions exist within the culture associating skin conditions with poor hygiene or sexual practices that go against cultural morés. Auxiliary considerations include the disease's difficult and costly management and social issues such as the limitations placed on marriage prospects for young women. Using a generic QOL instrument, the authors identified a significant impact of pemphigus on QOL and highlighted that beyond functional aspects of disease burden, the SF-36 also explores social domains and self-image issues, which are particularly relevant in pemphigus.

The QOL of patients with pemphigus vulgaris was also evaluated in one study of 27 German participants using a dermatology-specific instrument. ${ }^{12}$ The dermatology-specific Dermatology Life Quality Index (DLQI) ${ }^{13}$ score for the study cohort averaged $10.1 \pm 6.6$ compared with healthy individuals with a mean score of $0.5 \pm 1.1$, and the authors concluded that a diagnosis of pemphigus vulgaris conferred a large impairment in QOL. Patients with mucosal involvement were found to have a higher DLQI, averaging 10.4 \pm 7.3, compared with those patients with mucosal sparing who averaged $9.3 \pm 5.1$, indicating a poorer QOL. Itching was also associated with decreased QOL as patients with itch scored $11.5 \pm 7.5$ compared with those without itch who scored 7.9 \pm 4.8 . Similarly, burning was found to impair QOL, with patients scoring $10.8 \pm 6.6$. This is compared with those without burning, who scored almost half of that with an average DLQI of 5.5 \pm 5.7 . Possibly, due to the small cohort size, none of the results achieved statistical significance and notably $22.2 \%$ 
of patients felt that their QOL was not impaired by their illness at all. The mean DLQI for pemphigus was higher than the mean scores for similar blistering diseases, such as bullous pemphigoid $(6.92 \pm 3.80)$, as well as other dermatological pathologies, such as basal cell carcinoma (2.0 \pm 2.2$)$, but lower than that of atopic eczema (12.5 \pm 5.8$)$ - a comparison facilitated by the use of a skin-specific QOL instrument to compare severity.

A study reviewing the health status of 58 Italian inpatients undergoing treatment for pemphigus also demonstrated a significant decrease in QOL compared with the greater population. ${ }^{14}$ Participants were issued the SF-36, and the Institute for Personality and Aptitude Testing Anxiety Scale Questionnaire (ASQ) ${ }^{15}$ and Clinical Depression Questionnaire (CDQ). ${ }^{16}$ As expected, pemphigus had a larger impact on SF-36 scores compared with normative data. ${ }^{17}$ No significant difference was observed between patients with pemphigus vulgaris $(n=51)$ or pemphigus foliaceus $(n=7)$; however, patients with disease duration of 5 years or more, recent disease onset, disease that had worsened compared with the previous year, mucocutaneous involvement, higher anti-Dsg3 antibodies and those patients with more severe disease subjectively all had worse SF-36 scores. The ASQ and CDQ values demonstrated a strong, negative correlation with the SF-36 scores indicating that poorer QOL scores were associated with higher levels of anxiety and depression.

Another study in this area used generic and dermatology-specific QOL instruments in 126 pemphigus patients. ${ }^{18}$ Most patients had pemphigus vulgaris $(\mathrm{n}=112)$, although the cohort included patients with pemphigus foliaceus $(\mathrm{n}=10)$, paraneoplastic pemphigus $(\mathrm{n}=2)$, and IgA pemphigus $(\mathrm{n}=2)$. Patients admitted to an Italian dermatological institute were asked to complete the SF-36, Skindex-29, and the 12-item General Health Questionnaire (GHQ-12) ${ }^{19}$ to assess QOL with disease severity evaluated with the Ikeda index ${ }^{20}$ and Physician Global Assessment as well as autoantibody titers. Compared with normative scores for the general Italian population, the SF-36 and Skindex-29 scores of pemphigus patients demonstrated a strong impact of the disease across all QOL domains. Poorer SF-36 scores were patients aged 50 years or older and those with multiple comorbidities. Disease severity and female sex were significantly associated with poorer SF-36 and Skindex-29 scores, which had not been identified in previous QOL studies in pemphigus, although has been described in other dermatological conditions such as psoriasis. ${ }^{21} \mathrm{GHQ}$ positivity (suggesting probable minor nonpsychotic psychiatric conditions) was detected in $39.7 \%$ of participants compared with a general population prevalence of $10 \%$ to $12 \%$ and was significantly correlated with clinical severity as assessed by Ikeda score. Interestingly, patients with pemphigus foliaceus were found to have a poorer QOL than those with pemphigus vulgaris using the Skindex-29, with a significant difference in the symptom scale with $70 \%$ of pemphigus foliaceus patients GHQ positive; however there were only 10 pemphigus foliaceus patients in this study compared with 112 pemphigus vulgaris patients, and as this study was completed in an inpatient setting perhaps the pemphigus foliaceus patients were more severe at presentation. As no objective disease extent score, such as the Autoimmune Bullous Skin Disease Intensity Score (ABSIS) ${ }^{22}$ or Pemphigus Disease Activity Index (PDAI), ${ }^{23}$ was provided with this study, it is not known if this was the case. No correlation was found between Dsg antibody titers, clinical severity, or QOL scores.

All studies to date evaluating QOL in autoimmune bullous dermatoses have identified a significant burden of disease in this setting. These results have been obtained through the use of generic and dermatology-specific QOL instruments; however, no disease-specific QOL measure has yet been developed to assess QOL in autoimmune bullous disease.

In contrast, such a measure has been established for use in the inherited bullous dermatoses epidermolysis bullosa (EB): the QOL in EB (QOLEB) questionnaire. ${ }^{24}$ The QOLEB was 
devised using standardized methods for the development of a validated QOL instrument. This included an exhaustive item-generation process with $26 \mathrm{~EB}$ patients of various types and severities, their families, and experts in the field. A pilot questionnaire was devised and distributed to $130 \mathrm{~EB}$ patients and from the 115 questionnaires returned, the instrument was revised using various methods, including factor analysis, to a 17-item questionnaire. The QOLEB has been shown to be a statistically valid and reliable means to evaluate QOLEB, correlating well with existing QOL instruments and distinguishing EB subtypes by score. Most existing QOL measures, even dermatology-specific instruments, review QOL domains incompatible with the lifestyle of EB sufferers (eg, domestic duties, sexual function) and accordingly have significant content validity issues. These can result in an underrepresentation of the burden of disease in this condition, highlighting the importance of disease-specific QOL instruments that capture issues particular to the skin condition being studied. The QOLEB has been shown to have a high validity, specific enough to capture the QOL concerns particular to EB and broad enough to encompass the different subtypes of $\mathrm{EB}$, and has the potential to be a sensitive, disease-specific tool to assess QOLEB in future studies. The QOLEB is already undergoing international validation studies in various foreign languages and was used in a recent randomized controlled trial of cell therapy for recessive dystrophic $\mathrm{EB}(\mathrm{DEB}) .{ }^{25}$

QOLEB has also been reviewed using more generic tools to determine the burden of disease. A Scottish study of 116 EB patients, 75 EB simplex (EBS) patients, 40 DEB patients, and 1 patient with generalized severe recessive dystrophic EB (RDEB-gS), used the DLQI or the Children's Dermatology Life Quality Index (CDLQI) to assess QOL in this setting. ${ }^{26}$ Both adults and children with EBS or DEB were found to experience impairment in QOL akin to that of patients with moderate to severe psoriasis and atopic dermatitis and at the time of publication, no other dermatological condition was known to impinge greater on QOL than RDEB-gS. This impairment is further emphasized by the fact that patient responses were often artificially lowered because of the content validity discussed previously.

Another study administered questionnaire sets, including the SF-36, Skindex-29, GHQ-12, EuroQol 5 dimensions questionnaire (EQ-5D) or EQ-5D child, ${ }^{27}$ Family Strain Questionnaire (FSQ), ${ }^{28}$ and a 5-point Patient Global Assessment scale, ${ }^{29}$ to patients stratified by age. The study included the responses of 125 Italian EB patients: 18 EBS patients, 15 non-Herlitz junctional EB (JEB) patients, 83 DEB patients, and 9 patients with Kindler syndrome. Patients with EB reported low physical scale scores statistically lower than the general population and mental health scores were only slightly below normative values. No significant difference was observed among EB subtypes according to Skindex-29 scores; however, patients with JEB and severe generalized recessive DEB had lower General Health scores as part of their SF-36. Patients with higher perceived disease severity had significantly higher scores in all Skindex-29 scales. Adult women had higher SF-36 and Skindex-29 scores and GHQ-positivity was also significantly higher in women than men (46\% compared with 18\%). Family burden increased with body surface area involvement and patient perception of disease severity. Interestingly, the burden of disease in EBS was shown to be higher than anticipated, particularly when compared with other subtypes of EB such as DEB. As DEB is a lifelong dermatosis, an acceptance of significant disruption to normal functioning and the development of coping strategies may account for the resilience displayed within this cohort. In contrast, patients with EBS who ostensibly have greater potential for normal functioning may have a heightened sensitivity to the restrictions imposed by their lesions, leading to a more pronounced burden of disease for these patients.

Less formalized methods of assessing QOL have also been used in the setting of EB. A Dutch study used semistructured interviews with 11 children with EB as a qualitative means of evaluating QOL. ${ }^{30}$ The authors identified that the effects of EB on QOL could be broadly 
ordered into problems associated with itch, pain, difficulties engaging with others, the visibility of the disease, and feelings of being different. Another study assessed mobility, pain, and independence in ADLs as proxies for QOL in 140 children representative of the major subtypes of EB. ${ }^{31}$ All participants were shown to be at least partially dependent for some ADLs, especially those associated with walking and personal hygiene. Total independence during childhood was the norm in EBS and dominant DEB, whereas those with JEB and recessive DEB were likely to be totally dependent for at least some ADLs, as expected. Another study explored the psychosocial impact of EB on patients and carers by administering a standardized questionnaire to families with a child affected by EB. ${ }^{32}$ Divorce was common and usually attributed to the impact of the disease on the marriage. Additionally, $10 \%, 25 \%, 36 \%$, and $64 \%$ of parents of an affected child with EBS, dominant DEB, recessive DEB, and JEB respectively characterized their relationship as revolving almost exclusively around the care of their affected child, highlighting the need to recognize the burden on QOL for carers as well as patients, and consider counseling or respite care to preserve the family unit.

\section{Conclusions}

The impact of a blistering dermatosis on QOL is multifaceted and may impose a significant burden. Integrating formal evaluation of QOL into the clinical evaluation of patients facilitates an assessment of disease severity and mapping of disease trajectory, and can capture outcomes of therapeutic intervention relevant to the patient. Accordingly, the evaluation of QOL necessitates a validated methodology to accurately quantify the magnitude and the degree to which it impedes normal day-to-day functioning. QOL instruments serve this function and are promising tools for patient assessment in the future.

\section{References}

1. WHOQOL Group. The World Health Organization Quality of Life assessment (WHOQOL): position paper from the World Health Organization. Soc Sci Med. 1995; 41:1403-9. [PubMed: 8560308]

2. Both H, Essink-Bot M, Busschbach J, et al. Critical review of generic and dermatology-specific health-related quality of life instruments. J Invest Dermatol. 2007; 127:2726-39. [PubMed: 17989733]

3. Rapp S, Feldman S, Exum M, et al. Psoriasis causes as much disability as other major medical diseases. J Am Acad Dermatol. 1999; 41:401-7. [PubMed: 10459113]

4. Chen S. Dermatology quality of life instruments: sorting out the quagmire. J Invest Dermatol. 2007; 127:2726-39. [PubMed: 17989733]

5. Sampogna F, Picardi A, Melchi C, et al. The impact of skin diseases on patients: comparing dermatologist's opinions with research data collected on patients. Br J Dermatol. 2003; 148:989-95. [PubMed: 12786831]

6. VanBeek M, Beach S, Braslow L, et al. Highlights from the report of the working group on "Core measures of the burden of skin diseases. J Invest Dermatol. 2007; 127:2701-6. [PubMed: 18007688]

7. Wiebe S, Guyatt G, Weaver B, et al. Comparative responsiveness of generic and specific quality-oflife instruments. J Clin Epidemiol. 2003; 56:52-60. [PubMed: 12589870]

8. Finlay A, Khan G, Luscombe D, et al. Validation of sickness impact profile and psoriasis disability index in psoriasis. Br J Dermatol. 1990; 123:751-6. [PubMed: 2265090]

9. Ware J Jr, Sherbourne C, The MOS. 36-item short form health survey (SF-36). Conceptual framework and item selection. Med Care. 1992; 30:472-83.

10. Masahiro S, Shigaku I, Yutaka I, Ogawa H. An investigation of quality of life (QOL) of pemphigus patients in Japan (First report). J Dermatol. 2000; 110:283-8. 
11. Terrab Z, Benchikhi H, Maaroufi A, et al. Quality of life and pemphigus. Ann Dermatol Venereol. 2005; 132:321-8. [PubMed: 15886558]

12. Mayrshofer F, Hertl M, Sinkgraven R. Significant decrease in quality of life in patients with pemphigus vulgaris. Results from the German Bullous Skin Disease (BSD) Study Group. J Dtsch Dermatol Ges. 2005; 3:431-5. [PubMed: 15892845]

13. Finlay A, Khan G. Dermatology Life Quality Index (DLQI)—a simple practical measure for routine clinical use. Clin Exp Dermatol. 1994; 19:210-6. [PubMed: 8033378]

14. Tabolli S, Mozzetta A, Antinone V, et al. The health impact of pemphigus vulgaris and pemphigus foliaceus assessed using the Medical Outcomes Study 36-item short form health survey questionnaire. Br J Dermatol. 2008; 158:1029-34. [PubMed: 18294312]

15. Krug, S.; Scheier, I.; Cattel, R. Handbook for the IPAT Anxiety Scale. Champaign (IL): Institute for Personality and Ability Testing; 1976.

16. Krug, S.; Laughlin, J. Handbook for the IPAT Depression Scale. Champaign (IL): Institute for Personality and Ability Testing; 1976.

17. Sampogna F, Tabolli S, Soderfeldt B, et al. Measuring quality of life of patients with different clinical types of psoriasis using the SF-36. Br J Dermatol. 2006; 154:844-9. [PubMed: 16634884]

18. Paradisi A, Sampogna F, Di Pietro C, et al. Quality-of-life assessment in patients with pemphigus using a minimum set of evaluation tools. J Am Acad Dermatol. 2009; 60:26-9.

19. Goldberg, D.; Williams, PA. user's guide to the General Health Questionnaire. Windsor, UK: NFER-Nelson; 1988.

20. Ikeda S, Imamura S, Hashimoto I, et al. History of the establishment and revision of diagnostic criteria, severity index and therapeutic guidelines for pemphigus in Japan. Arch Dermatol Res. 2003; 295(Suppl):S12-6. [PubMed: 12677427]

21. Sampogna F, Chren M, Melchi C, et al. Age, gender, quality of life and psychological distress in patients hospitalised with psoriasis. Br J Dermatol. 2006; 154:325-31. [PubMed: 16433804]

22. Pfütze M, Niedermeier A, Hertl M, et al. Introducing a novel Autoimmune Bullous Skin Disease Intensity Score (ABSIS) in pemphigus. Eur J Dermatol. 2007; 17:4-11. [PubMed: 17324820]

23. Rosenbach M, Murrell DF, Bystryn JC, et al. Reliability and convergent validity of two outcome instruments in pemphigus. J Invest Dermatol. 2009; 129:2404-10. [PubMed: 19357707]

24. Frew JW, Martin LK, Nijsten T, et al. Quality of life evaluation in epidermolysis bullosa (EB) through the development of the QOLEB questionnaire: an EB-specific quality of life instrument. Br J Dermatol. 2009; 161:1323-30. [PubMed: 19681875]

25. Venugopal SS, Yan WF, Frew JW, et al. First double-blind randomized clinical trial of intradermal allogeneic fibroblast therapy for severe generalized recessive dystrophic epidermolysis bullosa randomized against placebo injections resulted in similar wound healing that is independent of collagen VII expression. J Investig Dermatol. 2010; 130:S67.

26. Horn HM, Tidman MJ. Quality of life in epidermolysis bullosa. Clin Exp Dermatol. 2002; 27:70710. [PubMed: 12472552]

27. Brooks, R.; Rabin, RE.; de Charro, F. The measurement and validation of health status using EQ-5D: a European perspective. Dordrecht: Kluwer Academic; 2005.

28. Ferrario SR, Baiardi P, Zotti AM. Update on Family Strain Questionnaire: a tool for the general screening of caregiving-related problems. Qual Life Res. 2004; 13:1425-34. [PubMed: 15503838]

29. Tabolli S, Sampogna F, Di Pietro C, et al. Quality of life in patients with epidermolysis bullosa. Br J Dermatol. 2009; 161:869-77. [PubMed: 19624546]

30. Scheppingen C, Lettinga AT, Duipmans JC, et al. Main problems experienced by children with epidermolysis bullosa: a qualitative study with semi-structured interviews. Acta Derm Venereol. 2008; 88:143-50. [PubMed: 18311442]

31. Fine JD, Johnson LB, Weiner M, et al. Assessment of mobility, activities and pain in different subtypes of epidermolysis bullosa. Clin Exp Dermatol. 2004; 29:122-7. [PubMed: 14987264]

32. Fine JD, Johnson LB, Weiner M, et al. Impact of inherited epidermolysis bullosa on parental interpersonal relationships, marital status and family size. Br J Dermatol. 2005; 152:1009-14. [PubMed: 15888161] 